\section{Diagnostik von Suchterkrankungen}

In den vergangenen 20 Jahren sind die theoretischen Konstrukte, mit denen Suchtphänomene diagnostisch abgebildet werden, wesentlich weiterentwickelt worden, ohne jedoch einen Standard zu erreichen, der der Komplexität des Geschehens in umfassendem Sinn Rechnung trägt. Mit deskriptiven Klassifikationssystemen erfolgt heute eine hinreichend zuverlässige kategoriale Zuordnung $\mathrm{zu}$ den wesentlichen diagnostischen Clustern, wie dies im Beitrag von Freyberger und Stieglitz dargestellt wird. Eine weitere Verbesserung der Zuverlässigkeit läßt sich vor allem durch den Einsatz von diagnostischen Instrumenten (vor allem Interviews) erreichen, dies gilt insbesondere, wenn es um die Erfassung von Komorbidität geht, wie im Beitrag von Stieglitz und Freyberger gezeigt wird. Auch die Verwendung komplexerer multiaxialer Systeme führt nicht daran vorbei, im Hinblick auf Ätiologie, Pathogenese, Symptomatologie, Krankheitsverlauf, Rehabilitation und Restitution jeweils hoch spezifische individuell variierende Konstellationen $\mathrm{zu}$ beschreiben. Die umstrittene Erweiterung des Suchtbegriffs auf nicht-stoffgebundene Phänomene hat darüber hinaus kaum zur Weiterentwicklung diagnostischer Konzepte beigetragen und eine gewisse Irritation verursacht. Hinzu kommt, dass die Therapierelevanz diagnostischer Konzepte in breiten Bereichen nicht hinreichend untersucht ist und sich mit der sich zunehmend diagnosenorientierten Vergütung von Therapien seitens der Kostenträger (u.a. DRG-Diskussion) eine empirisch wenig fundierte Strategie herauskristallisiert. Dies ist von nicht unerheblicher versorgungspolitischer Relevanz, weil tradierte standardisierte Interventionsformen allmählich von individualisierten, diversifizierten und flexiblen therapeutischen Angeboten abgelöst werden, die Trennung zwischen Akutversorgung und Rehabilitation zunehmend in Frage gestellt wird.

Generell ist festzustellen, dass bei den vorliegenden Verfahren im Alkoholbereich zahlreiche Instrumente bestehen, während für den Bereich der Medikamenten- oder Drogenabhängigkeit kaum psychometrisch überprüfte Methoden zu finden sind. Nach der Einschätzung von Schwoon und Krausz [1] ist dies zum einen darauf zurückzuführen, dass die Substanzen hinsichtlich Einnahme, Wirkungen, Folgeerscheinungen äußerst unterschiedlich sind und in ihrer Vielfalt nicht in umfassende Verfahren gebündelt werden können; zum anderen vor allem im Drogenbereich darauf, dass durch Illegalität und Strafandrohung die Bereitschaft zur Durchführung entsprechender Forschungsvorhaben und zur Mitwirkung daran gering war.

Eine wesentliche Entwicklung der letzten Jahre, die in diesem Heft nicht aufgegriffen wird, aber genannt werden soll, betrifft den Addiction Severity Index (ASI), der mittlerweile in

Suchttherapie 2001; 2: 1

(c) Georg Thieme Verlag Stuttgart · New York

ISSN 1439-9903
Harald J. Freyberger, Rolf-Dieter Stieglitz

der 5. Auflage vorliegt $[2,3]$. Hierbei handelt es sich um ein halbstrukturiertes Interviewverfahren, das sich auf alle Formen von Substanzmißbrauch und -abhängigkeit erstreckt und gleichermaßen für gruppenstatistische Querschnittsuntersuchungen, für Verlaufsuntersuchungen als auch für die individuelle Therapieplanung eignet. Zur Sicherung der internationalen Vergleichbarkeit wird eine Version gegenwärtig als Europ-ASI erprobt [4]. Die Anweisungen zur Durchführung der deutschsprachigen Version liegen in einem Manual vor [5].

Mit dem insgesamt zu konstatierenden steigenden Interesse an der Evaluation therapeutischer Fragestellungen ist darüber hinaus in den letzten Jahren das Bedürfnis gewachsen, über eine deskriptive Diagnostik hinaus, abhängigkeitsspezifische und therapeutisch relevante Merkmale genauer zu erfassen. Obgleich das in diesem Kontext zu erwähnende Craving-Konzept umstritten bleibt, sind in den vergangenen Jahren hier erhebliche Forschungsanstrengungen erfolgt, die die Autorengruppe um Veltrup et al. in diesem Heft darstellt.

Der zunehmende Kostendruck im Gesundheitswesen und die Internationalisierung von Forschungsansätzen sowie die Förderungs- und die Publikationspolitik werden dazu führen, dass verstärkt auf die internationale Vergleichbarkeit diagnostischer Instrumente geachtet werden wird.

Wie aber die Schwierigkeiten bei der transkulturellen Übertragung von Instrumenten deutlich machen, gelingt allein aus methodischer Sicht die Konstruktion von Tests gleicher faktorieller Validität selten überzeugend, selbst wenn die sprachliche Vergleichbarkeit einigermaßen gegeben ist [1]. Hier wird sich in den kommenden Jahren noch ein erheblicher Forschungsbedarf ergeben.

\section{Literatur}

${ }^{1}$ Schwoon DR, Krausz M. In: Stieglitz RD, Baumann U, Freyberger H.J (Hrsg).psychischer Störungen in der Klinischen Psychologie, Psychotherapie und Psychiatrie. Psychodiagnostik. Stuttgart: Thieme, 2001; (im Druck)

${ }^{2}$ McLellan AT, Loborsky L, Woody GE, O’Brien CP. An improved evaluation instrument for substance abuse patients: The Addiction Severity Index. Journal of Nervous and Mental Disease, 1980; 168: 26-33

${ }^{3}$ McLellan AT, Kushner H, Metzger D, Peters R, Smith I, Grissom G, Pettinati H, Argeriou M. The fifth edition of the Addiction Severity Index. Journal of Substance Abuse Treatment 1992; 9: 199-213

${ }^{4}$ Gsellhofer B. Der Addiction Severity Index: Überprüfung der psychometrischen Werte einer deutschsprachigen Version und Vergleich zwischen der deutschen und einer amerikanischen Stichprobe. Unveröffent. Dissertation (Psychologie). Universität Tübingen, 1998 